\title{
Isospin asymmetry and type-I superconductivity in neutron star matter
}

\author{
Mark Alford ${ }^{1}$, Gerald Good ${ }^{1}$, and Sanjay Reddy ${ }^{2}$ \\ ${ }^{1}$ Physics Department, Washington University, St. Louis, MO 63130, USA and \\ ${ }^{2}$ Theoretical Division, Los Alamos National Laboratory, Los Alamos, NM 87545, USA
}

(Dated: August 22, 2005)

\begin{abstract}
It has been argued by Buckley et. al. 1] that nuclear matter is a type-I rather than a type-II superconductor. The suggested mechanism is a strong interaction between neutron and proton Cooper pairs, which arises from an assumed $U(2)$ symmetry of the effective potential, which is supposed to originate in isospin symmetry of the underlying nuclear interactions. To test this claim, we perform an explicit mean-field calculation of the effective potential of the Cooper pairs in a model with a simple four-point pairing interaction. In the neutron star context, matter is very neutron rich with less than $10 \%$ protons, so there is no neutron-proton pairing. We find that under these conditions our model shows no interaction between proton Cooper pairs and neutron Cooper pairs at the mean-field level. We estimate the leading contribution beyond mean field and find that it is is small and attractive at weak coupling.
\end{abstract}

PACS numbers: 26.60.+c,74.20.-z,97.60.Jd

Recently, it has been suggested that nuclear matter in neutron stars might be a type-I superconductor. The astrophysical evidence is that certain neutron stars have long precession periods, and it has been suggested that this means the proton superconductivity cannot be type-II [2], although that inference has been contested [3, 44]. A theoretical argument for type-I superconductivity in neutron star cores was then presented by Buckley et. al. in Ref. [1]. These authors assumed that the effective potential for the Cooper pair fields has a $U(2)$ symmetry under rotation of proton and neutron Cooper pairs into each other. Specifically, the effective potential for the Cooper pair fields was assumed to take the form

$$
\begin{gathered}
V\left(\left|\Delta_{p p}\right|^{2},\left|\Delta_{n n}\right|^{2}\right) \approx U\left(\left|\Delta_{p p}\right|^{2}+\left|\Delta_{n n}\right|^{2}\right) \\
=-\mu_{c}\left(\left|\Delta_{p p}\right|^{2}+\left|\Delta_{n n}\right|^{2}\right)+a\left(\left|\Delta_{p p}\right|^{2}+\left|\Delta_{n n}\right|^{2}\right)^{2}+\cdots
\end{gathered}
$$

The assumption that the potential is approximately a function of $\left|\Delta_{p p}\right|^{2}+\left|\Delta_{n n}\right|^{2}$ leads to $\left|\Delta_{p p}\right|^{2}\left|\Delta_{n n}\right|^{2}$ cross terms, which provide a strong repulsive interaction between the neutron and proton Cooper pair condensates. It is argued in Ref. [1] that this leads to a long-range attraction between proton flux tubes, i.e. type-I superconductivity.

The assumed $U(2)$ symmetry was justified by invoking the isospin symmetry of the underlying nuclear interaction. The authors of Ref. 1] admit that isospin is severely broken by the constraint of electrical neutrality, which, combined with beta-equilibration, greatly suppresses the proton Fermi momentum relative to the neutron Fermi momentum, but they claim that this does not affect the interaction between the $p-p$ and $n-n$ Cooper pairs. This seems implausible, since it is well known in the theory of superconductivity that the coefficients of the terms in the Landau-Ginzburg effective theory, including the quartic coupling $a$, depend strongly on the Fermi momenta of the underlying fermions 5]. For example, for a one component Fermi gas, just below the BCS critical point, the effective Landau-Ginzburg potential is

$$
V\left(|\Delta|^{2}\right)=-\mu|\Delta|^{2}+a|\Delta|^{4}+\cdots
$$

where $\mu \approx N_{F}\left(T_{c}-T\right) / T_{c}$, and $N_{F}$ is the density of states at the Fermi surface, so for non-relativistic fermions $N_{F} \propto m p_{F}$ where $m$ is the mass and $p_{F}$ is the Fermi momentum. The minimum of the potential occurs at $\Delta_{0}=$ $\sqrt{\mu /(2 a)}$, so the quartic coefficient is $a=\mu /\left(2 \Delta_{0}^{2}\right)$. The binding energy of the condensate is then $V\left(\Delta_{0}\right)=-\frac{1}{2} N_{F} \Delta_{0}^{2}$, a well-known result in the superconductivity literature [6]. We see that $\mu, a$, and $V\left(\Delta_{0}\right)$ are all very sensitive to the Fermi momenta of the underlying fermions. Clearly for protons and neutrons in a neutron star, which have very different Fermi momenta but similar pairing condensates $\Delta$, this will not give an effective potential of the form (11).

We now back up this argument with a concrete calculation. We work with a very simple microscopic model for nucleon-nucleon interactions, and analyze the pairing using the mean-field approximation. We take into account the requirements of electrical neutrality and equilibration under the weak interactions, which disfavor neutron-proton pairing. At zero temperature the potential is not accurately described by an low-order polynomial like (11), but we can expand around the mean field and obtain the quartic coupling between small fluctuations $\phi_{n n}, \phi_{p p}$,

$$
\Omega\left(\phi_{p p}, \phi_{n n}\right)=\cdots+\alpha_{p p} \phi_{p p}^{4}+\alpha_{n n} \phi_{n n}^{4}+\alpha_{n p} \phi_{p p}^{2} \phi_{n n}^{2}+\cdots
$$

By explicitly calculating these couplings we find that they do not obey the $U(2)$ symmetry assumed in Ref. [1]. In fact, in the mean field approximation our result (14) shows no interaction at all between the proton and neutron Cooper 
pairs: $\alpha_{n p}=0$. This appears to be a generic mean-field result, and does not depend on any specific features of the pairing interaction. Further, and as expected from the preceding discussion, we find that $\alpha_{p p} \ll \alpha_{n n}$. We then discuss the lowest-order corrections beyond the mean-field approximation by calculating the effective interaction between neutron and proton Cooper pairs diagrammatically. We show that this interaction is sub-leading in the coupling and is negligible in weak coupling. At temperatures close to the critical temperature, where Landau-Ginzburg theory can be used to analyze vortex structure, we find that the interaction between the $p p$ condensate and the $n n$ is weak and repulsive, not strong and attractive as Eq. (10) would imply.

Our simple model of neutrons and protons is based on the isospin-symmetric Lagrangian

$$
\begin{aligned}
\mathcal{L} & =\mathcal{L}_{\text {kinetic }}+\mathcal{L}_{\text {int }}, \\
\mathcal{L}_{\text {kinetic }} & =N_{\alpha a}^{\dagger}\left(\frac{\partial}{\partial \tau}-\frac{\nabla^{2}}{2 m}-\mu_{a}\right) N_{\alpha a}, \\
\mathcal{L}_{\text {int }} & =-\frac{G}{2}\left(N_{\alpha a}^{\dagger} N_{\alpha a}\right)^{2} .
\end{aligned}
$$

The nucleon field $N_{a \alpha}$ has isospin index $a=n, p$ and spin index $\alpha=\uparrow, \downarrow$. Repeated indices are summed. We immediately generalize the interaction to allow different couplings for protons and neutrons, and Fierz-transform it into the pairing form. We assume that all pairing is in the rotationally invariant $s$-wave channel, so keeping only those terms we obtain

$$
\begin{aligned}
\mathcal{L}_{\text {int }} & =-G_{p p} p_{\uparrow}^{\dagger} p_{\downarrow}^{\dagger} p_{\downarrow} p_{\uparrow}-G_{n n} n_{\uparrow}^{\dagger} n_{\downarrow}^{\dagger} n_{\downarrow} n_{\uparrow} \\
& -\frac{1}{2} G_{n p}\left(p_{\uparrow}^{\dagger} n_{\downarrow}^{\dagger}+n_{\uparrow}^{\dagger} p_{\downarrow}^{\dagger}\right)\left(n_{\downarrow} p_{\uparrow}+p_{\downarrow} n_{\uparrow}\right) .
\end{aligned}
$$

The isospin-symmetric case corresponds to $G_{p p}=G_{n n}=G_{n p}$. This interaction will lead to pairing of the fermions at their Fermi surfaces, by the usual BCS mechanism. We can calculate the thermodynamic potential of the paired state by a standard Hubbard-Stratonovich transformation that introduces complex bosonic Cooper-pair fields $\Delta_{n n}, \Delta_{p p}$, and $\Delta_{p n}$ (for a review, see [7]). The Lagrangian then has three parts: the kinetic term is as before, and the others contain the Cooper pair fields:

$$
\begin{aligned}
\mathcal{L}= & \mathcal{L}_{\text {kinetic }}+\mathcal{L}_{\Delta}+\mathcal{L}_{F}, \\
\mathcal{L}_{\Delta}= & \left|\Delta_{n n}\right|^{2} / G_{n n}+\left|\Delta_{p p}\right|^{2} / G_{p p}+\left|\Delta_{p n}\right|^{2} / G_{n p}, \\
\mathcal{L}_{F}= & -\Delta_{n n}^{*} n_{\downarrow} n_{\uparrow}-\Delta_{p p}^{*} p_{\downarrow} p_{\uparrow} \\
& -\frac{1}{\sqrt{2}} \Delta_{p n}^{*}\left(p_{\downarrow} n_{\uparrow}+n_{\downarrow} p_{\uparrow}\right)+\text { h.c. }
\end{aligned}
$$

By adjusting the phases of the $n$ and $p$ fields we can choose $\Delta_{n n}$ and $\Delta_{p p}$ to be real, leaving $\Delta_{p n}$ complex. As is well known [8], in neutron star matter the mismatch between the proton and neutron Fermi surfaces completely suppresses $n-p$ pairing, so $\Delta_{p n}=0$ even at small isospin asymmetry, but for now we keep the $\Delta_{p n}$ term. In the mean-field approximation, we neglect any space-time variation in the $\Delta$ fields and set them equal to their vacuum expectation values. We can then integrate out the fermions, giving us the volume density of the thermodynamic potential or grand canonical potential $\Omega=E / V-\mu N / V=-p$. In the rest of this paper we will loosely refer to this as the "thermodynamic potential". We find

$$
\Omega=\mathcal{L}_{\Delta}-\int \frac{d \omega}{2 \pi} \int^{\Lambda} \frac{d^{3} k}{(2 \pi)^{3}} \ln \operatorname{Det} M,
$$

where we have introduced an ultraviolet cutoff $\Lambda$ on the three-dimensional momentum integral. To obtain this expression, we wrote the inverse propagator in the Nambu-Gork'ov basis $\left(p_{\uparrow}, n_{\uparrow}, p_{\downarrow}^{\dagger}, n_{\downarrow}^{\dagger}, p_{\downarrow}, n_{\downarrow}, p_{\uparrow}^{\dagger}, n_{\downarrow}^{\dagger}\right)$, and observed that it block-diagonalizes into two identical $4 \times 4$ blocks,

$$
M=\left(\begin{array}{cccc}
-i \omega+\epsilon_{p} & 0 & -\Delta_{p p} & -\frac{\Delta_{p n}}{\sqrt{2}} \\
0 & -i \omega+\epsilon_{n} & -\frac{\Delta_{p n}}{\sqrt{2}} & -\Delta_{n n} \\
-\Delta_{p p} & -\frac{\Delta_{p n}^{*}}{\sqrt{2}} & -i \omega-\epsilon_{p} & 0 \\
-\frac{\Delta_{p n}^{*}}{\sqrt{2}} & -\Delta_{n n} & 0 & -i \omega-\epsilon_{n}
\end{array}\right) .
$$


Note that when $\Delta_{p n}=0, M$ further decomposes into $2 \times 2$ blocks, for $\left(p_{\uparrow}, p_{\downarrow}^{\dagger}\right),\left(n_{\uparrow}, n_{\downarrow}^{\dagger}\right)$ etc, which describe the $s$-wave pairing of protons and neutrons respectively. In Eq. [8], $\epsilon_{p} \equiv k^{2} / 2 m-\mu_{p}$ and $\epsilon_{n} \equiv k^{2} / 2 m-\mu_{n}$. The determinant is straightforward to compute and we obtain

$$
\begin{aligned}
\operatorname{Det} M & =\left(\omega^{2}+\epsilon_{n}^{2}\right)\left(\omega^{2}+\epsilon_{p}^{2}\right)+\Delta_{n n}^{2}\left(\omega^{2}+\epsilon_{p}^{2}\right) \\
& +\Delta_{p p}^{2}\left(\omega^{2}+\epsilon_{n}^{2}\right)+\left|\Delta_{p n}\right|^{2}\left(\omega^{2}+\epsilon_{p} \epsilon_{n}\right) \\
& +\left(\Delta_{n n} \Delta_{p p}-\Delta_{p n}^{2} / 2\right)\left(\Delta_{n n} \Delta_{p p}-\Delta_{p n}^{* 2} / 2\right)
\end{aligned}
$$

The requirement that the vacuum expectation values $\Delta_{p p}, \Delta_{n n}$, and $\Delta_{p n}$ minimize the thermodynamic potential is expressed in the three gap equations,

$$
\begin{aligned}
\frac{\Delta_{n n}}{G_{n n}} & =\int \frac{d \omega d^{3} k}{(2 \pi)^{4}} \frac{\Delta_{n n}\left(\omega^{2}+\epsilon_{p}^{2}\right)+\Delta_{p p}\left(\Delta_{p p} \Delta_{n n}-\operatorname{Re} \Delta_{p n}^{2} / 2\right)}{\operatorname{det} M} \\
\frac{\Delta_{p p}}{G_{p p}} & =\int \frac{d \omega d^{3} k}{(2 \pi)^{4}} \frac{\Delta_{p p}\left(\omega^{2}+\epsilon_{n}^{2}\right)+\Delta_{n n}\left(\Delta_{p p} \Delta_{n n}-\operatorname{Re} \Delta_{p n}^{2} / 2\right)}{\operatorname{det} M} \\
\frac{\Delta_{p n}}{G_{n p}} & =\int \frac{d \omega d^{3} k}{(2 \pi)^{4}} \frac{\Delta_{p n}\left(\omega^{2}+\epsilon_{p} \epsilon_{n}\right)-\Delta_{p n}^{*}\left(\Delta_{p p} \Delta_{n n}-\Delta_{p n}^{2} / 2\right)}{\operatorname{det} M}
\end{aligned}
$$

When $\Delta_{p n}=0$, as in neutron star matter [8] these equations decouple into independent gap equations for $\Delta_{p p}$ and $\Delta_{n n}$.

Because our model interaction is so simple, it predicts pairing gaps that rise with the density of states near the Fermi surface, so $\Delta$ rises with $p_{F}$. This means that it does not produce realistic neutral nuclear matter, in which the interaction is isospin-symmetric, and $p_{F p} \ll p_{F n}$ (from electrical neutrality) but nevertheless $\Delta_{p p} \approx \Delta_{n n}$. In the real world, this happens because the nuclear interaction at short distance is repulsive, so even though $p_{F n} \gg p_{F p}$, the neutrons end up with a similar pairing gap to the protons. In our simple model, which is strictly valid only at low density, the four-Fermion coupling only encodes the attractive part of the interaction through the s-wave scattering length. We will choose $G_{p p}$ to be larger than $G_{n n}$ so that in the neutral system the protons and neutrons have the same pairing gap, as in real nuclear matter. It will turn out that our essential conclusion, that there is no $U(2)$ symmetry of the effective potential for the Cooper pair fields, holds irrespective of whether or not the the couplings $G_{p p}$ and $G_{n n}$ are equal. It is then reasonable to guess that $G_{n p}$ should have a value somewhere between $G_{n n}$ and $G_{p p}$. To be specific, we shall employ typical values $\mu_{n} \sim 60 \mathrm{MeV}\left(k_{F n} \sim 335 \mathrm{MeV}\right)$ and $\mu_{p} \sim 8 \mathrm{MeV}\left(k_{F p} \sim 123 \mathrm{MeV}\right)$ and a momentum cut-off $\Lambda=750 \mathrm{MeV}$. For these parameters the four-fermion couplings that gives $\Delta_{n n} \sim 1 \mathrm{MeV}$ and $\Delta_{p p} \sim 1 \mathrm{MeV}$ are $G_{n n} \sim 1 \times 10^{-5} \mathrm{MeV}^{2}$ and $G_{p p} \sim 2 \times 10^{-5} \mathrm{MeV}^{2}$, respectively.

Since $\Delta_{n p}=0$ in beta-equilibrated neutral nuclear matter, we can write the mean-field thermodynamic potential

$$
\begin{aligned}
\Omega & =\frac{\Delta_{n n}^{2}}{G_{n n}}+\frac{\Delta_{p p}^{2}}{G_{p p}}-\int \frac{d \omega}{2 \pi} \int^{\Lambda} \frac{d^{3} k}{(2 \pi)^{3}}\left(\ln \left(\omega^{2}+\epsilon_{n}^{2}+\Delta_{n n}^{2}\right)+\ln \left(\omega^{2}+\epsilon_{p}^{2}+\Delta_{p p}^{2}\right)\right) \\
& =\frac{\Delta_{n n}^{2}}{G_{n n}}-\int^{\Lambda} \frac{d^{3} k}{(2 \pi)^{3}}\left(\sqrt{\epsilon_{n}^{2}+\Delta_{n n}^{2}}-\epsilon_{n}\right)+\frac{\Delta_{p p}^{2}}{G_{p p}}-\int^{\Lambda} \frac{d^{3} k}{(2 \pi)^{3}}\left(\sqrt{\epsilon_{n}^{2}+\Delta_{n n}^{2}}-\epsilon_{p}\right)
\end{aligned}
$$

We see that this is equal to the sum of the thermodynamic potentials for two species of Cooper pair bosons that do not interact with each other. There are no cross-terms between $\Delta_{p p}$ and $\Delta_{n n}$. The effective potential does not take the form (11). This result did not depend on the specific form of the interaction. It simply arises from the fact that beta-equilibrium and electrical neutrality require $p_{F p} \ll p_{F n}$, and Cooper pairing is suppressed between species with very different Fermi momenta.

We may use Eq. (12) to investigate the nature of the quartic terms that describe the coupling between fluctuations in the Cooper-pair densities. Fluctuations in the pairing field $\phi_{n n}, \phi_{p p}$ are defined through the following substitutions in Eq. (12): $\Delta_{n n} \rightarrow \tilde{\Delta}_{n n}+\phi_{n n}$ and $\Delta_{p p} \rightarrow \tilde{\Delta}_{p p}+\phi_{p p}$ where $\tilde{\Delta}_{n n}$ and $\tilde{\Delta}_{p p}$ are the ground state expectation values that satisfy the gap equations. For small fluctuations an expansion of the thermodynamic potential about the mean field ground state is well motivated. Retaining only the quartic terms

$$
\Omega\left(\phi_{p p}, \phi_{n n}\right)=\cdots+\alpha_{p p} \phi_{p p}^{4}+\alpha_{n n} \phi_{n n}^{4}+\alpha_{n p} \phi_{p p}^{2} \phi_{n n}^{2}+\cdots .
$$

From the preceding discussion it is clear that in the mean field approximation there are no cross-terms. The coefficients $\alpha_{n n}$ and $\alpha_{p p}$ are non zero and depend in general on the chemical potentials, $\tilde{\Delta}_{n n}$ and $\tilde{\Delta}_{p p}$. Explicitly, by Taylor 


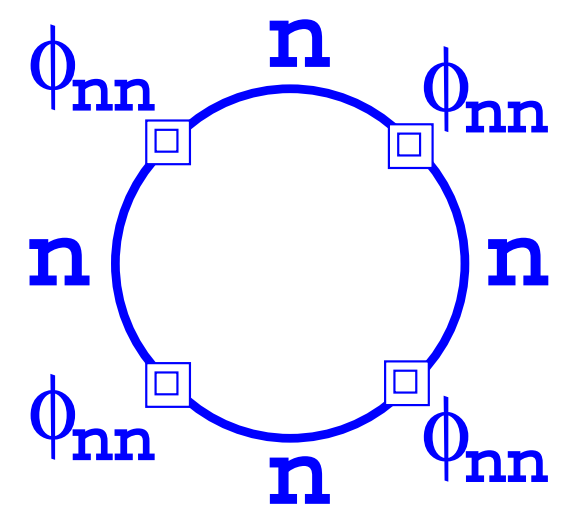

FIG. 1: The lowest-order mean-field contribution to $\alpha_{n n}$, the coefficient of the $\phi_{n n}^{4}$ term in the effective potential, which describes scattering between low-momentum fluctuations $\phi_{n n}$ in the $n n$ condensate. The thick lines are Nambu-Gork'ov neutron propagators. Each double-square vertex is an insertion of the $\phi_{n n}$ operator. There is a similar diagram for $\alpha_{p p}$.

expanding Eq. (12) we find

$$
\begin{aligned}
& \alpha_{n p}=0 \\
& \alpha_{n n}=\frac{1}{8} \int \frac{d^{3} k}{(2 \pi)^{3}} \frac{1}{E_{n}^{3}}-\frac{6 \tilde{\Delta}_{n n}^{2}}{E_{n}^{5}}+\frac{5 \tilde{\Delta}_{n n}^{4}}{E_{n}^{7}}, \\
& \alpha_{p p}=\frac{1}{8} \int \frac{d^{3} k}{(2 \pi)^{3}} \frac{1}{E_{p}^{3}}-\frac{6 \tilde{\Delta}_{p p}^{2}}{E_{p}^{5}}+\frac{5 \tilde{\Delta}_{p p}^{4}}{E_{p}^{7}},
\end{aligned}
$$

where $E_{n}=\sqrt{\epsilon_{n}^{2}+\tilde{\Delta}_{n n}^{2}}$ and $E_{p}=\sqrt{\epsilon_{p}^{2}+\tilde{\Delta}_{p p}^{2}}$. These contributions can also be calculated in a diagrammatic approach using the Nambu-Gorkov Greens functions [9]. The diagram for $\alpha_{n n}$ is shown in Fig. [1 and there is an analogous one for $\alpha_{p p}$. We find

$$
\alpha_{n n}=\frac{1}{4} \int \frac{d^{3} k}{(2 \pi)^{3}} k T \sum_{s} \operatorname{Tr}\left[\mathcal{G}_{n}\left(k, i \omega_{s}\right) \tau \mathcal{G}_{n}\left(k, i \omega_{s}\right) \tau \mathcal{G}_{n}\left(k, i \omega_{s}\right) \tau \mathcal{G}_{n}\left(k, i \omega_{s}\right) \tau\right]
$$

where the finite temperature Nambu-Gorkov Green's function for the neutron superfluid, expressed in the $\left(n_{\uparrow}, n_{\downarrow}^{\dagger}\right)$ basis (see text after Eq. [8]), is

$$
\mathcal{G}_{n}\left(k, i \omega_{s}\right)=\frac{1}{\left(i \omega_{s}\right)^{2}-E_{n}^{2}(p)}\left(\begin{array}{cc}
-i \omega_{s}+\epsilon_{n} & -\Delta_{n n} \\
-\Delta_{n n} & -i \omega_{s}-\epsilon_{n}
\end{array}\right),
$$

and insertions of the fluctuating pairing field are

$$
\tau=\left(\begin{array}{ll}
0 & 1 \\
1 & 0
\end{array}\right)
$$

The Matsubara frequency is $\omega_{s}=(2 s+1) \pi k T$. In evaluating these diagrams we ignore any momentum transfer since we are interested only in the low momentum fluctuations. We have explicitly checked that this gives the same result as Eq. (14). At zero temperature and when $\tilde{\Delta} / \mu$ is small we obtain the following analytic expressions

$$
\begin{aligned}
\alpha_{n n} & =-\frac{M k_{F n}}{24 \pi^{2} \tilde{\Delta}_{n n}^{2}}\left(1+\mathrm{O}\left[\frac{\tilde{\Delta}_{n n}^{2}}{\mu_{n}^{2}}\right]\right), \\
\alpha_{p p} & =-\frac{M k_{F p}}{24 \pi^{2} \tilde{\Delta}_{p p}^{2}}\left(1+\mathrm{O}\left[\frac{\tilde{\Delta}_{p p}^{2}}{\mu_{p}^{2}}\right]\right) .
\end{aligned}
$$

We see that the couplings are proportional to the corresponding Fermi momenta, $\alpha_{n n} \propto k_{F n}$ and $\alpha_{p p} \propto k_{F p}$, so the scattering of proton condensate fluctuations is much weaker than that of neutron condensate fluctuations, indicating 


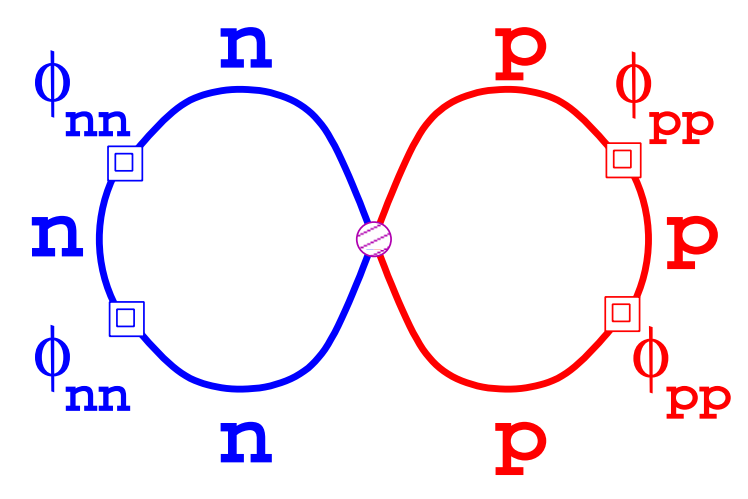

FIG. 2: The lowest-order contributions beyond mean field to $\alpha_{n p}$, the coefficient of the $\phi_{n n}^{2} \phi_{p p}^{2}$ term in the effective potential, which describes scattering between low-momentum fluctuations $\phi_{n n}$ and $\phi_{p p}$ in the $n n$ and $p p$ condensates. The thick lines are Nambu-Gork'ov neutron or proton propagators. The double square vertices are insertions of the $\phi_{n n}$ and $\phi_{p p}$ operators. The hatched vertex is the fundamental $n^{\dagger} n p^{\dagger} p$ interaction in the Lagrangian.

a strong breaking of any symmetry that rotates proton Cooper pairs into neutron Cooper pairs. Further, the sign of $\alpha_{n n}$ and $\alpha_{p p}$ is negative. However, since we are Taylor expanding about the global minimum of the thermodynamic potential, there are lower and higher order terms (not explicitly written in Eq. (13)) that ensure that the system is stable with respect to both small and large fluctuations. This is in contrast to the usual Landau- Ginzburg analysis just below $T_{c}$, where a Taylor expansion about the normal state is characterized by a negative quadratic coefficient and a positive quartic term.

We now discuss corrections to Eq. (12) beyond the mean field approximation. The leading order diagram that contributes to the scattering between neutron and proton fluctuations is shown in Fig. 2 The important point is that this diagram involves the fundamental four-fermion neutron-proton interaction $G_{n p}$. Evaluating the diagram, we find that the leading-order beyond-mean-field contribution to the effective four-point interaction between the proton and neutron condensate fluctuations is

$$
\begin{aligned}
\alpha_{n p}= & \frac{1}{4} G_{n p} \int \frac{d^{4} k}{(2 \pi)^{4}} \operatorname{Tr}\left[\mathcal{G}_{n}(k, i \omega) \tau \mathcal{G}_{n}(k, i \omega) \tau \mathcal{G}_{n}(k, i \omega) \tau_{3}\right] \\
& \times \int \frac{d^{4} k}{(2 \pi)^{4}} \operatorname{Tr}\left[\mathcal{G}_{p}(k, i \omega) \tau \mathcal{G}_{p}(k, i \omega) \tau \mathcal{G}_{p}(k, i \omega) \tau_{3}\right] \\
= & -G_{n p} \frac{k_{F n}^{3} k_{F p}^{3}}{64 \pi^{4} \mu_{n}^{2} \mu_{p}^{2}} f\left(\frac{\Delta_{n n}}{\mu_{n}}\right) f\left(\frac{\Delta_{p p}}{\mu_{p}}\right),
\end{aligned}
$$

where $\mathcal{G}_{n}(k, i \omega)$ and $\mathcal{G}_{p}(k, i \omega)$ are the Nambu-Gorkov Green's functions for the neutrons and protons respectively, and $\tau_{3}=\operatorname{diag}(1,-1)$ is the diagonal Pauli matrix [9]. The dimensionless function $f$ is defined by the integral

$$
f(\delta)=\int_{-1}^{\infty} d x x \sqrt{x+1} \frac{x^{2}-2 \delta^{2}}{\left(\delta^{2}+x^{2}\right)^{5 / 2}} .
$$

For $\delta \ll 1$ we obtain the following analytic relation

$$
f(\delta)=-1+\log \left[\frac{8}{\delta}\right]+\mathcal{O}\left[\delta^{2}\right]
$$

To see whether beyond-mean-field corrections can raise $\alpha_{n p}$ to a value comparable to $\alpha_{n n}$ or $\alpha_{p p}$ we follow Ref. [1] in defining an asymmetry parameter

$$
\epsilon=\left(\alpha_{n n} \alpha_{p p}-\alpha_{n p}^{2}\right) /\left(\alpha_{n n} \alpha_{p p}\right) .
$$

(Ref. 1] expresses $\epsilon$ in terms of the quartic couplings $a_{p p}, a_{n n}, a_{n p}$ for the expansion around $\Delta=0$, but it should be equally valid to expand around the mean field, which is the minimum of the thermodynamic potential.) Ref. [1] concluded that type-I superconductivity requires $\epsilon \lesssim 1 / 20$. As we have seen, in the mean-field approximation $\epsilon=1$. From Eq. (21) we find that the beyond-mean-field corrections to $\epsilon$ are negligible by several orders of magnitude. $\epsilon$ is always within $10^{-4}$ of unity. 
All our calculations so far have been at zero temperature, in which case the expansion of the potential around the mean-field ground state is only valid for small deviations from that state. This makes it impossible to discuss the expected structure of the vortices, in which the pairing fields vary from zero to their vacuum values. In order to be able to say anything about the vortices, we have to work at temperatures close to the critical temperature $T_{c}$, where a traditional Landau-Ginzburg analysis is possible, using an expansion around the zero-mean-field state with only the quadratic and quartic terms,

$$
\Omega\left(T \sim T_{c} ; \phi_{n n}, \phi_{p p}\right)=-\mu_{n n}^{c} \phi_{n n}^{2}-\mu_{p p}^{c} \phi_{p p}^{2}+a_{p p}^{c} \phi_{p p}^{4}+a_{n n}^{c} \phi_{n n}^{4}+a_{n p}^{c} \phi_{p p}^{2} \phi_{n n}^{2}+\cdots .
$$

We shall now perform such an analysis. We assume that the critical temperatures of the neutrons and protons are not vastly different and we perturb about the Fermi gas ground state. The diagrammatic method described earlier permits us to calculate the coefficients in Eq. (25). Since we are perturbing about the normal state, we use the Green's function defined in Eq. (16) but with $\Delta_{n n}=\Delta_{p p}=0$. The quadratic coefficient

$$
\begin{aligned}
& \mu_{n n}^{c}=\frac{1}{G_{n n}}+\Pi_{n n}, \text { where } \Pi_{n n}=\frac{1}{2} \int \frac{d^{3} k}{(2 \pi)^{3}} k T \sum_{s} \operatorname{Tr}\left[\mathcal{G}_{n}^{0}\left(k, i \omega_{s}\right) \tau \mathcal{G}_{n}^{0}\left(k, i \omega_{s}\right) \tau\right] \\
& \mu_{p p}^{c}=\frac{1}{G_{p p}}+\Pi_{p p}, \text { where } \Pi_{p p}=\frac{1}{2} \int \frac{d^{3} k}{(2 \pi)^{3}} k T \sum_{s} \operatorname{Tr}\left[\mathcal{G}_{p}^{0}\left(k, i \omega_{s}\right) \tau \mathcal{G}_{p}^{0}\left(k, i \omega_{s}\right) \tau\right]
\end{aligned}
$$

where $\mathcal{G}_{n}^{0}\left(k, i \omega_{s}\right)=\lim _{\Delta_{n} \rightarrow 0} \mathcal{G}_{n}\left(k, i \omega_{s}\right)$ and $\mathcal{G}_{p}^{0}\left(k, i \omega_{s}\right)=\lim _{\Delta_{p} \rightarrow 0} \mathcal{G}_{p}\left(k, i \omega_{s}\right)$. An explicit calculation yields

$$
\begin{aligned}
& \mu_{n n}^{c}=\frac{1}{G_{n n}}-\frac{(2 m k T)^{3 / 2}}{8 \pi^{2} k T} J_{1}\left(\frac{\mu_{n}}{k T}\right), \\
& \mu_{p p}^{c}=\frac{1}{G_{p p}}-\frac{(2 m k T)^{3 / 2}}{8 \pi^{2} k T} J_{1}\left(\frac{\mu_{p}}{k T}\right),
\end{aligned}
$$

where the dimensionless integral

$$
J_{1}(\gamma)=\int_{0}^{\tilde{\Lambda}} d x \frac{\sqrt{x}}{x-\gamma} \tanh \left[\frac{x-\gamma}{2}\right],
$$

and $\tilde{\Lambda}=\Lambda^{2} /(2 m k T)$. The critical temperature $T_{c}$ for neutron and protons is defined by the relation $\mu_{n n}^{c}=0$ and $\mu_{p p}^{c}=0$, respectively. As expected, for $T>T_{c}$ we obtain $\mu_{n n}^{c}, \mu_{p p}^{c}>0$ while $\mu_{n n}^{c}, \mu_{p p}^{c}<0$ for $T<T_{c}$. The quartic coefficient is

$$
a_{n n}^{c}=\frac{1}{4} \int \frac{d^{3} k}{(2 \pi)^{3}} k T \sum_{s} \operatorname{Tr}\left[\mathcal{G}_{n}^{0}\left(k, i \omega_{s}\right) \tau \mathcal{G}_{n}^{0}\left(k, i \omega_{s}\right) \tau \mathcal{G}_{n}^{0}\left(k, i \omega_{s}\right) \tau \mathcal{G}_{n}^{0}\left(k, i \omega_{s}\right) \tau\right]
$$

and $a_{p p}^{c}$ is given by the same relation but with the $\mathcal{G}_{p}^{0}\left(k, i \omega_{s}\right)$ replacing $\mathcal{G}_{n}^{0}\left(k, i \omega_{s}\right)$. The Matsubara sum is easily evaluated and we obtain

$$
a_{n n}^{c}=\frac{(2 m k T)^{3 / 2}}{64 \pi^{2}(k T)^{3}} J_{3}\left(\frac{\mu_{n}}{k T}\right)
$$

where

$$
J_{3}(\gamma)=\int_{0}^{\tilde{\Lambda}} d x \frac{\sqrt{x}}{(x-\gamma)^{3}} \frac{\sinh [x-\gamma]-(x-\gamma)}{(1+\cosh [x-\gamma])}
$$

The coefficient that couples between neutron and proton pairing fields is given by

$$
a_{n p}^{c}=-\frac{G_{n p} m^{3}}{32 \pi^{4}(k T)} J_{2}\left(\frac{\mu_{n}}{k T}\right) \times J_{2}\left(\frac{\mu_{p}}{k T}\right),
$$

where

$$
J_{2}(\gamma)=\int_{0}^{\tilde{\Lambda}} d x \frac{\sqrt{x}}{(x-\gamma)^{2}} \frac{\sinh [x-\gamma]-(x-\gamma)}{(1+\cosh [x-\gamma])}
$$


Thus in the vicinity of the critical temperature the asymmetry parameter $\epsilon$ can be estimated using $a_{n n}^{c}, a_{p p}^{c}$ and $a_{n p}^{c}$ calculated above. For our choice of parameters we find that $T_{c} \simeq 0.6 \mathrm{MeV}$. For $T=0.4 \mathrm{MeV}$ the coefficients of the effective potential are $\mu_{n n}^{c}=5520 \mathrm{MeV}^{2}, \mu_{p p}^{c}=2010 \mathrm{MeV}^{2}, a_{n n}^{c}=2627, a_{p p}^{c}=968$ and $a_{n p}^{c}=-3\left(G_{n p} / G_{n n}\right)$. As before, the asymmetry parameter $\epsilon \simeq 1$ since $a_{n p} \ll a_{n n}$ and $a_{n p} \ll a_{p p}$. The fact that $a_{n p}^{c}$ is negative implies that the neutron(proton) superfluid density will decrease in the inner core of the proton(neutron) vortex.

Our conclusion is that in neutral nuclear matter, the disparity between the neutron and proton Fermi momenta provides a strong explicit breaking of the $U(2)$ symmetry posited in Ref. 1]. This breaking is far too strong to allow the proposed mechanism for type-I superconductivity to operate. Although a calculation of the vortex structure is beyond the scope of this work, our leading order calculation of $a_{n p}^{c}$ provides a means to estimate the strength of the effective interaction between neutron and proton condensate vortices, at temperatures close to $T_{c}$. We note however that due to the non-perturbative nature of the interaction between nucleons we cannot exclude the possibility of a strong coupling between the neutron and proton superfluids. In our simple model the attractive interaction between neutrons and protons directly leads to a negative $a_{n p}^{c}$ leading to a depletion of the neutron superfluid in the core of the proton vortex. This result is robust as long as the effective interaction between neutrons and protons is attractive and contradicts the predictions in Ref. [1] where the neutron superfluid density increased inside the proton vortex.

\section{Acknowledgments}

We acknowledge helpful conversations with T. Battacharya, M. Metlitski, G. Rupak, and A. Zhitnitsky. The work of MGA and GG is supported by the U.S. Department of Energy under grant number DE-FG01-91ER40628. The work of SR is supported by the U.S. Department of Energy under grant number W-7405-ENG-36.

[1] K. B. W. Buckley, M. A. Metlitski and A. R. Zhitnitsky, Phys. Rev. Lett. 92, 151102 (2004) arXiv:astro-ph/0308148; Phys. Rev. C 69, 055803 (2004) arXiv:hep-ph/0403230.

[2] B. Link, Phys. Rev. Lett. 91, 101101 (2003) arXiv:astro-ph/0302441.

[3] P. B. Jones, Phys. Rev. Lett. 92, 149001 (2004).

[4] A. Sedrakian, arXiv:astro-ph/0408467

[5] A. L. Fetter and J. D. Walecka, "Quantum Theory of Many-Particle Systems", McGraw-Hill, 1971.

[6] M. Tinkham, "Introduction to Superconductivity", 2nd ed., (McGraw-Hill, 1996). Sections (3.1) and (3.4.2).

[7] M. Stone, "The Physics of Quantum Fields", Springer, New York, 2000.

[8] A. Sedrakian and U. Lombardo, Phys. Rev. Lett. 84, 602 (2000) arXiv:nucl-th/9907076. U. Lombardo, P. Nozieres, P. Schuck, H. J. Schulze and A. Sedrakian, Phys. Rev. C 64, 064314 (2001) arXiv:nucl-th/0109024]. M. Baldo, U. Lombardo, H. J. Schulze and Z. Wei, Phys. Rev. C 66, 054304 (2002). D. J. Dean and M. Hjorth-Jensen, Rev. Mod. Phys. 75, 607 (2003) arXiv:nucl-th/0210033.

[9] J. R. Schrieffer, "Theory of Superconductivity", Perseus Books, 3rd ed., Reading, 1983. 